EPJ Web of Conferences 82, 01036 (2015)

DOI: $10.1051 /$ epjconf/20158201036

(C) Owned by the authors, published by EDP Sciences, 2015

\title{
Determination of the critical conditions of heat transfer in a LED
}

\author{
Oleg M. Rudenko and Pavel A. Strizhak
}

National Research Tomsk Polytechnic University 30, Lenin Avenue, Tomsk 634050, Russia

\begin{abstract}
The mathematical and physical models of heat transfer were developed in order to determine the critical conditions of heat transfer in typical light-emitting diodes (LED). Operating time of LEDs under mentioned critical conditions and typical heating temperature of a compound were calculated.
\end{abstract}

\section{Introduction}

LEDs are widely used in different industry areas. At first sight, this semiconductor seems to be simple, but the involved thermal processes demand additional attention and understanding of specialists. Many research works are related to the processes of LEDs [1-4], but it is still difficult to identify a complete evaluative model of heat transfer. Normally, the determination of critical conditions of the heat exchange is achieved experimentally. Each type of LEDs requires a specific type of experiments and needs previous estimations or calculations.

The reasons of LEDs' failures as well as deterioration of initial characteristics at temperature above $373 \mathrm{~K}$ demand additional attention. Some materials of LEDs' components can withstand temperatures above $2000 \mathrm{~K}$ [5] while other materials lose their initial characteristics and features at temperatures above 400-500 K [6]. This means that critical conditions for some elements of LEDs (especially for a compound) can be from 0,1 to $1 \mathrm{~ms}$.

For the calculation of reliability indicators and critical conditions of the heat transfer a thermal model [1] and calculations based on it are used in most cases. Normally, the calculation of a thermal model is connected with the calculation of the thermal resistance and patterns of change of the volt-ampere characteristics depending on the time and temperature [2,3].

One of the main characteristics of the LED is the direct current flowing through the crystal. When a current flows through the crystal (through the PN-junction) it is important to determine the critical conditions of heat exchange in the area of a compound near the crystal since the bandwidth of the light flux depends on the temperature of the compound.

The aim of this work is to make a mathematical simulation of the heat transfer processes for determination of the critical conditions of the heat exchange in the LED.

This is an Open Access article distributed under the terms of the Creative Commons Attribution License 4.0, which permits unrestricted use, distribution, and reproduction in any medium, provided the original work is properly cited. 


\section{Model of the heat transfer}

In order to illustrate the physical model, the scheme view of a typical LED is shown in Fig. 1.

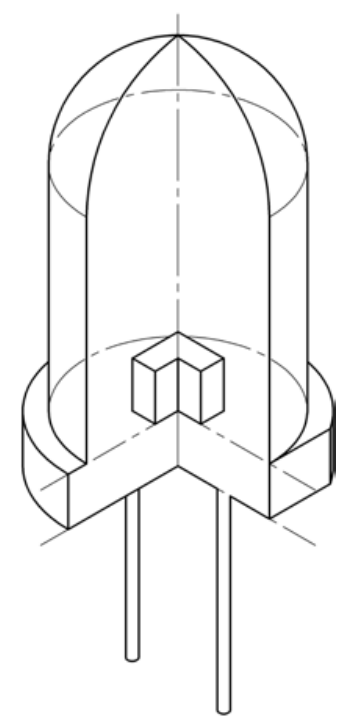

Figure 1. Scheme view of a typical LED.

Before formulating the aim of this research it was assumed that the current flowing through the crystal exceeds the operating current 20-40 times. The heating of the crystal takes place in a short time, after that the heat is transferred to the compound and the substrate. It was assumed that the compound is filled with a homogeneous medium with unchanging thermo-physical properties over time. Since the melting point of the crystal and substrate are above $2000 \mathrm{~K}$ [5], the critical condition will be the heating of the compound's area near the crystal. It should be taken into account that all other mechanisms of heat transfer except radiation and heating were excluded. Figure 2 shows the scheme of heat transfer which is used in the simulation.

The main aim of the research was a determination of the critical conditions of the heat exchange in a LED at the maximum value of the currents. The solution of the problem of mutual processes of heat transfer taking place in the scheme "substrate - crystal - compound - lens - air" is described in [7].

\section{Data and results}

The melting point of the compound is considered to be at $450 \mathrm{~K}$. The temperature at the boundaries of the field of solutions is equal to $313 \mathrm{~K}$. The gallium nitride was taken as a crystal material while the silicon carbide was chosen as a material of a substrate. The environmental conditions are air and for the research purposes the silicon compound DowCorningOE-6636 (similar in its characteristics to epoxy resin) was taken. For the lens material an organic glass was selected. The thermo-physical characteristics are taken from $[5,6,8]$ and are summarized in Table 1.

In order to identify the critical conditions of the heat exchange the main focus should be put on the current flowing through the crystal. Melting temperatures can be achieved with the help of the heat exchange in the compound areas near to the crystal. Varying current of the crystal can help to determine the critical time at which the compound starts to melt (see Fig. 3). 


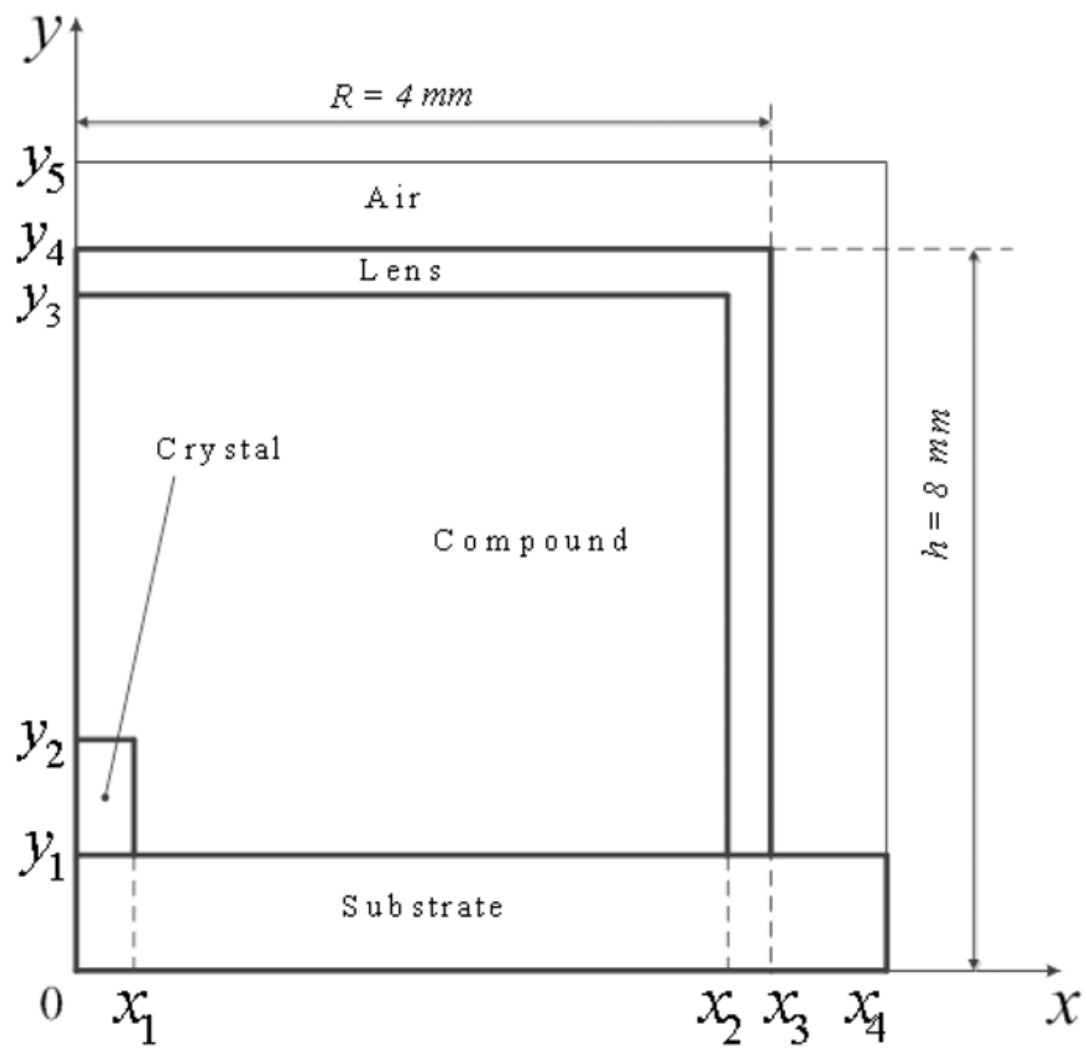

Figure 2. Scheme for heat transfer problem solving.

Table 1. Thermo physical characteristics of the main LED elements.

\begin{tabular}{|l|c|c|c|}
\hline Medium (material) & Heat capacity, J/(kg.K) & Density, $\mathrm{kg} / \mathrm{m}^{3}$ & Thermal conductivity, W/(m·K) \\
\hline Crystal (Gallium nitride) & 452 & 6150 & 130 \\
\hline Compound (Epoxy resin) & 1300 & 1160 & 0,2 \\
\hline Substrate (Silicon carbide) & 675 & 3160 & 490 \\
\hline Lens (Plexiglas) & 1467 & 1190 & 0,19 \\
\hline Air & 1,006 & 1190 & 0,026 \\
\hline
\end{tabular}

As it can be seen in Fig. 3, significant unsteady heating of the crystal and its high heat release in the border areas of the compound caused by the impulsive increase of the current value can be the critical condition of the heat exchange. The time at which the defects can occur in the areas of the compound vary from $0,013 \mathrm{sec}$ to $0,005 \mathrm{sec}$ at the current value $0,5 \mathrm{~A}$ and $1,0 \mathrm{~A}$ respectively. The operating time of modern LEDs indicated by the manufacturers is greater than 50000 hours, but if the critical conditions of the heat exchange are achieved even once, the operating time of the semiconductor can be reduced.

In order to prevent a rapid temperature increase in modern LEDs, different cooling systems and heat sinks materials $[9,10]$ are used. But even the use of such tools cannot always provide full protection against overheating. Accordingly, it is necessary to investigate as much as possible the process of heat exchange inside a LED under the effects of different factors. Since the experiment demands significant financial investments, it is necessary to develop models of heat and mass transfer and only after testing it use real devices. 


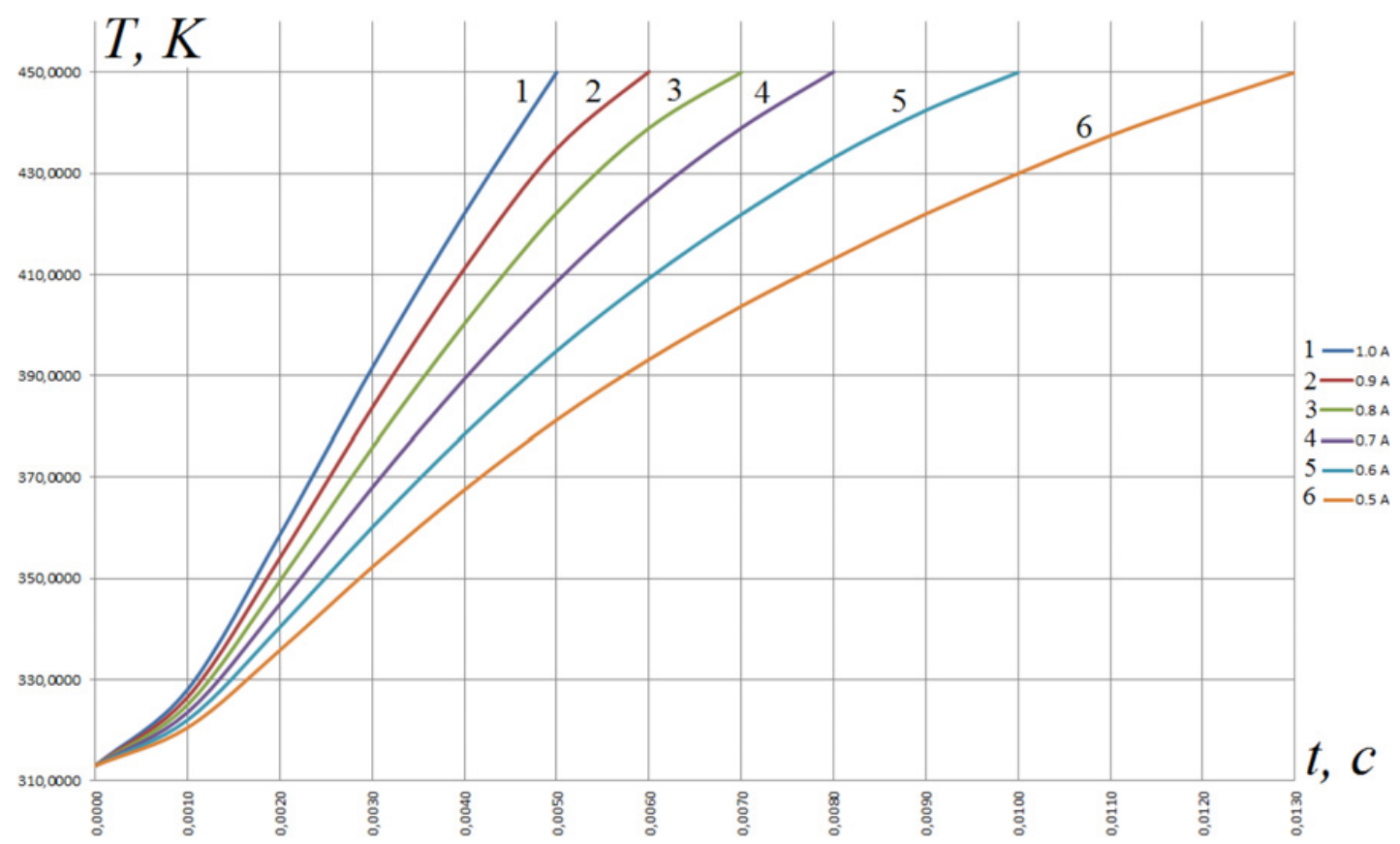

Figure 3. Scheme for solving the problem of heat transfer area.

\section{Conclusion}

The developed model of heat tranfer can determine critical conditions of heat exchange with the changing indicators of current flowing through the crystal and can also describe the conditions that influece other processes of heat and mass tranfer.

This work was financially supported by the government grant "Science" (project No. 2.1321.2014).

\section{References}

[1] M. Arik, C.A. Becker, S.E. Weaver, J. Petroski, SPIE, 5187, 64 (2004)

[2] V. Vigh, L. Kalmar, T. Regert, Bulletin of the Transilvania University of Brasov Series I: Eng., 6, 19 (2013)

[3] A.L. Zakgeim, G.L. Kuryshev, M.N. Mizerov, V.G. Polovinkin, I.V. Rozhansky, A.E. Chernyakov. Semiconductors, 44, 373 (2010)

[4] Chen, A. Keppens, P. Hanselaer, Y. Lu, Y. Gao, R. Zhuang, Z. Chen, Semiconductors, 46, 1310 (2012)

[5] Yu. Goldberg, M.E. Levinshtein, S.L. Rumyantsev, M.S. Shur, Properties of Advanced Semiconductor Materials: GaN, AIN, SiC, BN, SiC, SiGe (John Wiley \& Sons, Inc., New York, 2001)

[6] W.F. Smith, J. Hashemi, Foundations of Materials Science and Engineering, 5th edition, (McGraw-Hill, New York, 2010) 
[7] P.A. Strizhak, O.M. Rudenko, EPJ Web of Conferences, 76, 01022 (2014)

[8] Y.S. Touloukian, C.Y. Ho, Thermophysical Properties of Matter (Plenum Press, New York, 1972)

[9] D. Jang, S.-J. Yook, K.-S. Lee, Applied Energy, 116, 260 (2014)

[10] J.-H. Xiang, C.-1. Zhang, F. Jiang, X.-C. Liu, Y. Tang, Transactions of Nonferrous Metals Society of China, 21, 2066 (2011) 